\title{
An Associated Research for Genetic Polymorphism of 5-HTTLPR with Post-Traumatic Stress Disorder
}

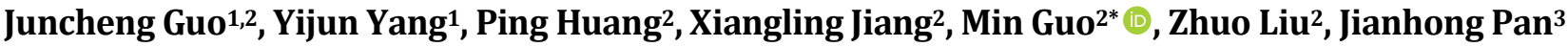 \\ ${ }^{1}$ Xiangya School of Medicine Affiliated Haikou Hospital, Central South University, Haikou, China \\ ${ }^{2}$ Department of Psychology, Hainan General Hospital, China \\ ${ }^{3}$ Shanghai Jiao Tong University, Shanghai, China \\ Email: *g2002m@163.com
}

How to cite this paper: Guo, J.C., Yang, Y.J., Huang, P., Jiang, X.L., Guo, M., Liu, Z. and Pan, J.H. (2019) An Associated Research for Genetic Polymorphism of 5-HTTLPR with Post-Traumatic Stress Disorder. Journal of Behavioral and Brain Science, 9, 1-12.

https://doi.org/10.4236/jbbs.2019.91001

Received: December 20, 2018

Accepted: January 8, 2019

Published: January 11, 2019

Copyright (๑) 2019 by author(s) and Scientific Research Publishing Inc. This work is licensed under the Creative Commons Attribution International License (CC BY 4.0).

http://creativecommons.org/licenses/by/4.0/

\begin{abstract}
The aim of this study was to investigate the influence of a polymorphism in the serotonin transporter gene (5-HTTLPR) in patients diagnosed with posttraumatic stress disorder (PTSD) in a Chinese sample of earthquake survivors. Polymerase chain reaction (PCR) amplification and amplified fragment length polymorphism (AFLP) were performed to type 5-HTTLPR promoter polymorphism in 57 PTSD patients and an equal number of healthy controls. The genotype and allele frequency distribution were analyzed and compared using various statistical methods. The frequency of LL, SL and SS genotypes in patients was found to be 5,16 and 36 respectively, in comparison to 16,22 and 19 in healthy controls. Fewer patients tended to be L genotype (22.8\%) than controls (47.4\%), but the number of patients with the S genotype was higher $(77.2 \%)$ compared to controls $(52.6 \%)$. The results show a statistically significant difference in genotype and allele frequency distribution between patients and controls. This research suggests that PTSD symptoms are significantly associated with 5-HTTLPR genetic polymorphism. These results add to the important research of genetics of psychiatric disorders, particularly in a Chinese context that has not been previously studied.
\end{abstract}

\section{Keywords}

Posttraumatic Stress Disorder, Gene Polymorphism, 5-HTTLPR, Genetics

\section{Introduction}

In recent years, the incidence of Post-Traumatic Stress Disorder (PTSD) has significantly increased due to the frequency of occurrence of natural and man-made 
disasters worldwide [1]. According to the diagnostic manual of the American Psychiatric Association, PTSD is a psychiatric disorder that can occur in people who have experienced or witnessed a traumatic event such as a natural disaster, a serious accident, a terrorist act, war/combat, rape, or other violent personal assault [2]. The 4 key symptoms of PTSD include: intrusion symptoms associated with the traumatic event(s); persistent avoidance of stimuli associated with the traumatic event(s) [3]; negative alterations in cognitions and mood associated with the traumatic event(s); and, marked alterations in arousal and reactivity associated with the traumatic event(s) [4]. Because of the higher incidence of PTSD after encountering a traumatic event [5] and the accompanying possibilities of serious detriments to the individual, family and society, this condition has become a popular topic for psychiatric and clinical psychological research in recent years [6]. With the increase of natural disasters occurring among the world and in China, (including earthquakes in Sichuan, Qinghai, Gansu and Yunnan), the incidence of psychological sequelae and more serious condition of PTSD has been increasing in recent years [7]. Therefore, exploring the mechanism of PTSD is important and necessary, to identify effective methods of prevention and treatment [8].

Most biological studies of PTSD have focused on changes in the gray matter nuclei and brain cortex, although recent research interest is being focused on genetics and immunology [9]. Of particular interest is the polymorphism in the serotonin transporter gene (5-HTTLPR) that has been studied in relation to both PTSD and depression [10], as the serotonin transporter (5-HTT) is responsible for terminating serotonin action in the synapsis. Interestingly, the human genetic polymorphism 5-HTTLPR results in a combination of short (S) and long (L) forms. The $S$ genotype restricts the transcriptional activity producing low functional expression of the 5-HTT and thus decreasing serotonin reuptake, while the L genotype does not results in this changes [11]. While current evidence does not fully support a direct effect of the polymorphism 5-HTTLPR on PTSD [12], the present study further aims at exploring the correlation of genotype and allele frequencies of 5-HTTLPR with PTSD in a Chinese population of earthquakes survivors. The study further explores a scientific basis for the clinical diagnosis of PTSD patients based on genetic research of the condition.

\section{Material and Methods}

\subsection{Subjects}

All cases in the study group were patients diagnosed with PTSD in the Hainan Province of China during the period from December 2013 to August 2014. This province was selected because the individuals living there had experienced a tragic earthquake several years before. The inclusion criteria were that patients were: 1) aged 35 to 55 years old; 2) right-handed; 3) met the diagnostic criteria for PTSD according to the Diagnostic and Statistical Manual of Mental Disorders, 5th Edition (DSM-5). Patients were excluded if they had a dual diagnosis of 
mental retardation, personality disorders, substance dependence or alcohol dependence. Voluntary and informed consent to participate in the research was obtained. In terms of their demographics, the study group consisted of 28 males and 29 females, with a mean age of $46.26( \pm 6.40)$ years, and mean years of schooling of $10.59( \pm 3.20)$ years. The control group consisted of subjects who did not meet any DSM-5 criteria for psychiatric disorder that were recruited. Specifically, the control group consisted of 30 males and 27 females, with a mean age of $42.92( \pm 5.98)$ years, and mean years of schooling of $10.96( \pm 3.08)$ years.

\subsection{Methods}

Conventional chlorine phenol extraction of genomic human DNA samples was used blood. The PCR primers used for genotyping were: forward

5'-GGCGTTGCCGCTCTGAATGC-3', reverse 5'-GAGGGACTGAGCTGGACAACCAC-3'. PCR was performed using the $E_{X}$ Taq DNA Polymerase reaction (Takara Clontech, BaoBiotechnology) and the PCR product was run in a $6 \%$ non-denaturing polyacrylamide gel vertically. Ethidium bromide (EB) detects electrophoresis images of each sample and determines the genotype by using UV gel analysis after staining (Figure 1(a), Figure $1(b))$.

5-HTTLPR gene polymorphism detection report (Table 1, Table 2, Table 3)

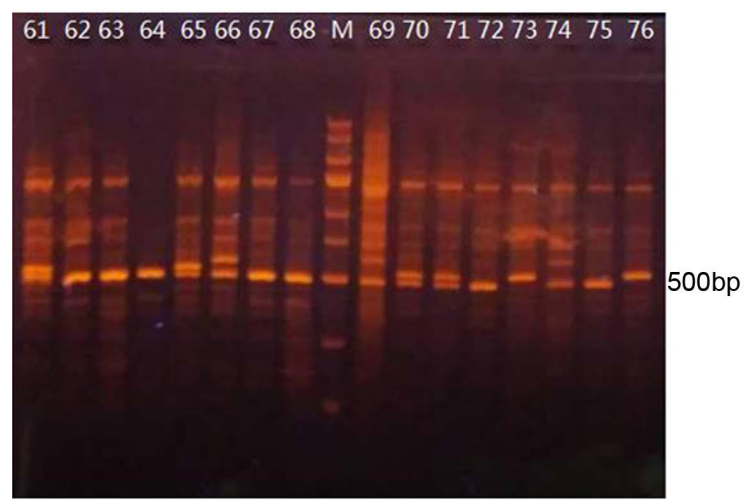

(a)

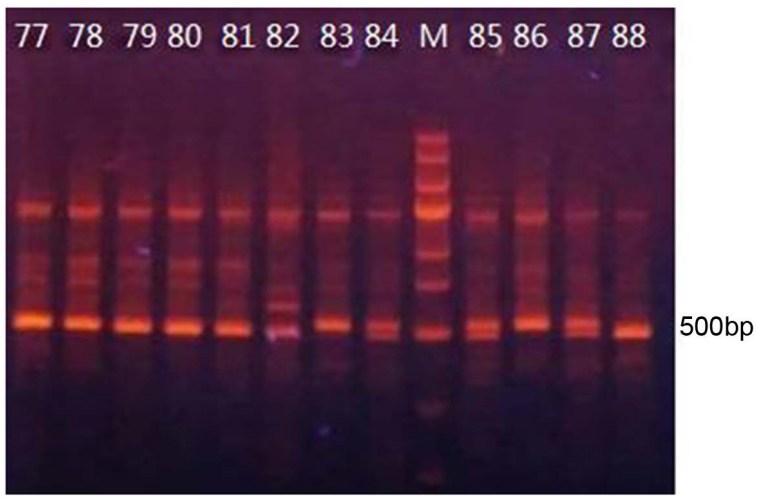

(b)

Figure 1. Electrophoresis result map. 
Table 1. PCR reaction system.

\begin{tabular}{cc}
\hline 2 mM dNTP & $1.5 \mathrm{ul}$ \\
$10 \times$ KOD buffer & $1.5 \mathrm{ul}$ \\
MgSO 4 & $0.6 \mathrm{ul}$ \\
DMSO & $1.5 \mathrm{ul}$ \\
Primer 5-HTT-F: $(10 \mathrm{uM})$ & $1 \mathrm{ul}$ \\
Primer 5-HTT-R: $(10 \mathrm{uM})$ & $1 \mathrm{ul}$ \\
template & $1 \mathrm{ul}$ \\
Blend Taq-Plus & $0.5 \mathrm{ul}$ \\
ddH2O & $6.4 \mathrm{ul}$ \\
Total & $15 \mathrm{ul}$ \\
\hline
\end{tabular}

Table 2. PCR amplification conditions.

\begin{tabular}{|c|c|}
\hline $94^{\circ} \mathrm{C} 2 \mathrm{~min}$ & \\
\hline $94^{\circ} \mathrm{C} 20 \mathrm{sec}$ & 25 \\
\hline $60^{\circ} \mathrm{C} 30 \mathrm{sec}$ & \\
\hline $72^{\circ} \mathrm{C} 40 \mathrm{sec}$ & \\
\hline $72^{\circ} \mathrm{C} 10 \mathrm{~min}$ & \\
\hline $4^{\circ} \mathrm{C}$ save & \\
\hline
\end{tabular}

Table 3. Statistical results.

(a)

\begin{tabular}{|c|c|c|}
\hline Result type & Sample number & $\begin{array}{l}\text { Number of } \\
\text { samples (a) }\end{array}$ \\
\hline SS type (484/484 bp) & 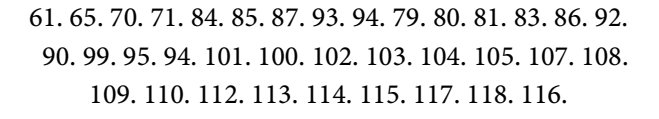 & 36 \\
\hline LL type $(528 / 528 \mathrm{bp})$ & 73. 76. 77. 78.91. & 5 \\
\hline SL type $(484 / 528 \mathrm{bp})$ & 62. 63. 64. 66. 67. 68. 69. 72. 74. 75. 82. 88. 89.96.97.98. & 16 \\
\hline
\end{tabular}

(b)

\begin{tabular}{ccc}
\hline Result type & Sample number & $\begin{array}{c}\text { Number of } \\
\text { samples (a) }\end{array}$ \\
\hline SS type $(484 / 484 \mathrm{bp})$ & 1.2 .3 .4 .5 .6 .7 .8 .9 .10 .11 .12 .13 .14 .15 .16 .17 .18$. & 19 \\
LL type $(528 / 528 \mathrm{bp})$ & 20.21 .23 .22 .24 .25 .26 .27 .28 .29 .30 .31 .32 .33 .34 .35$. & 16 \\
SL type $(484 / 528 \mathrm{bp})$ & 36.37 .38 .39 .40 .41 .42 .43 .44 .45 .46 .47 .48 .49 .50$. & 22 \\
\hline
\end{tabular}

First: reagents and instruments

1) Reagents: EasyPure ${ }^{\circledR}$ Genomic DNA Kit; Blend Taq-Plus BTQ-201

2) Instrument: Hema 9600 TE Thermo cycler

Second: the experimental process:

1) Blood sample genomic DNA extraction

a) Prepare a $56^{\circ} \mathrm{C}$ water bath in advance 
i) Place $200 \mathrm{ul}$ of blood sample in a sterile $1.5 \mathrm{ml}$ centrifuge tube.

ii) Add $100 \mu \mathrm{l}$ of LB2 and $20 \mu \mathrm{l}$ of Proteinase K.

iii) Incubate at $55^{\circ} \mathrm{C}$ until complete lysis (3 hours, 2 - 3 times per hour). Add $20 \mu \mathrm{l}$ of RNase A to the sample and incubate for 2 minutes at room temperature to remove RNA.

iv) Centrifuge at $12,000 \mathrm{rpm}$ for 5 minutes and transfer the supernatant to a sterile centrifuge tube.

b) Add $500 \mu \mathrm{l}$ of BB2, vortex for 5 seconds, and incubate for 10 minutes at room temperature.

c) Add all the solution to the spin column, centrifuge at $12,000 \mathrm{rpm}$ for 30 seconds, and discard the effluent.

d) Add $500 \mu \mathrm{l}$ of solution CB2, centrifuge at 12,000 rpm for 30 seconds, and discard the effluent.

e) Repeat step 4 once.

f) Add $500 \mu \mathrm{l}$ of solution WB2 (check for the addition of absolute ethanol before use), centrifuge at 12,000 rpm for 30 seconds, and discard the effluent.

g) Repeat step 6 once.

h) Centrifuge at 12,000 rpm for 2 minutes to completely remove residual WB2.

i) Place the spin column in a clean centrifuge tube, add $50-200 \mu$ preheated EB $\left(65^{\circ} \mathrm{C}\right)$ in the center of the column, or deionized water $(\mathrm{pH}>7.0)$. Allow to stand at room temperature for 1 minute, centrifuge at $12,000 \mathrm{rpm}$. In minutes, elute the DNA.

j) For more DNA, perform a second elution, add $50-200 \mu \mathrm{l}$ pre-heated EB $\left(65^{\circ} \mathrm{C}\right)$ in the center of the column, or deionized water $(\mathrm{pH}>7.0)$ for 1 minute at room temperature, 12,000 rpm Centrifuge for 1 minute and elute the DNA. The eluted DNA was stored at $-20^{\circ} \mathrm{C}$.

2) PCR and agarose gel electrophoresis identification

a) Using the extracted DNA as a template

b) Primer: 5-HTT-F: 5'-GGCGTTGCCGCTCTGAATGC-3',

5-HTT-R: 5'-GAGGGACTGAGCTGGACAAAC-3'.

Three: Experimental result.

\subsection{Statistical Analysis}

The data comparing the genotype and allele frequencies of two groups was analyzed using the SPSS13.0 statistical software package using the following statistical methods: Hardy-Weinberg (HW) goodness of fit test, frequency analysis, analysis of variance, $t$ test, and $\chi^{2}$ test. $p<0.05$ was used as the threshold for significance.

\section{Results}

\subsection{The Comparison between the Study Group and the Control Group}

The source of the research object is mainly in Hainan Province, a large number 
of immigrants from earthquake areas (Sichuan, Qinghai, Gansu and Yunnan) (the resettlement personnel around the Yangtze River Three Gorges Dam in China) settled in Hainan Province, passing our hospital. Patients in the psychological clinic were selected for PTSD; the source of the control group was mainly from immigrants from the earthquake-stricken areas to those who settled in Hainan Province. All participants in the study were willing and informed consent. In terms of demographics, there were no statistical differences in age, gender, and education between the study group and the control group.

As shown in Table 4, the demographic characteristics of gender, age and level of education of the study group (with PTSD) and the control group revealed no statistically significant differences $(p>0.05)$.

\subsection{Hardy-Weinberg Equilibrium Law Genetic Testing}

According to the Hardy-Weinberg equilibrium law, the distribution of the 5-HTTLPR gene is consistent with this genetic equilibrium law with a group representation. Table 5 shows the frequency of LL, SL and SS genotypes in the study group and control subjects. Specifically, patients were found to be 5,16 and 36 respectively, in comparison to 16, 22 and 19 in healthy controls. The study and control group test results showed that the mean difference was not significant $(p>0.05)$.

\subsection{Comparison of Genotypes and Allele between the Study Group and the Control Group}

Table 6 shows the comparison of frequencies of genotypes and allele between the study group and the control group. The table shows that the 5-HTTLPR gene in the PTSD study group had frequencies of $5,16,36$ respectively in the LL, SL and SS types. In comparison, the control group revealed frequencies of 16, 22, 19 respectively in the LL, SL and SS types. This resulted in a mean difference of 11.96, which it is statistically significant $(p=0.003)$. Table 6 also shows the allele frequency of the two groups, and reveals that for the PTSD group, the L gene appeared in $22.8 \%$ of cases and the $S$ gene appeared in $77.2 \%$ of cases. In contrast, for the control group, the L gene appeared in $47.4 \%$ of cases, and the $\mathrm{S}$ gene appeared in $52.6 \%$ of cases. The table shows that a comparison of these frequencies for the study and control group on the allele frequency revealed a mean difference of $15.10(p<0.01)$. Thus, both groups showed statistically significant differences between the genotypes and allele frequencies (see Table 6).

Table 4. Comparison of demographic characteristics of the study group and the control group.

\begin{tabular}{ccccc}
\hline \multirow{2}{*}{ Groups } & \multicolumn{2}{c}{$\mathrm{n}$} & Age (years) & $\begin{array}{c}\text { Years of education } \\
\text { (years) }\end{array}$ \\
\cline { 2 - 3 } & Male & Female & & $46.26 \pm 6.40$ \\
\hline The Study Group (PTSD) & 28 & 29 & & $10.59 \pm 3.20$ \\
The control group & 30 & 27 & $42.92 \pm 5.98$ & $10.96 \pm 3.08$ \\
$P$ & $\chi^{2}>0.05$ & $\chi^{2}>0.05$ & $t>0.05$ & $t>0.05$ \\
\hline
\end{tabular}


Table 5. The Hardy-Weinberg equilibrium law genetic testing of 5-HTTLPR gene distribution.

\begin{tabular}{cccccc}
\hline \multirow{2}{*}{ Groups } & \multicolumn{3}{c}{ Genotypes } & \multirow{2}{*}{$\chi^{2}$} & $P$ \\
\cline { 2 - 4 } & LL & SL & SS & & \\
\hline The Study Group (PTSD) & 5 & 16 & 36 & 2.34 & $>0.05$ \\
The control group & 16 & 22 & 19 & 2.91 & $>0.05$ \\
\hline
\end{tabular}

Table 6. The comparison of Genotypes and allele between study group and control group comparison [Frequency (\%)].

\begin{tabular}{ccccccc}
\hline \multirow{2}{*}{ Groups } & & \multicolumn{3}{c}{ Genotype frequencies (\%) } & \multicolumn{2}{c}{ Allele frequency (\%) } \\
\cline { 3 - 7 } & & LL & SL & SS & L & S \\
\hline The Study Group (PTSD) & 57 & $5(8.8)$ & $16(28.1)$ & $36(63.1)$ & $26(22.8)$ & $88(77.2)$ \\
The control group & 57 & $16(28.1)$ & $22(38.6)$ & $19(33.3)$ & $54(47.4)$ & $60(52.6)$ \\
$\chi^{2}$ & & & 11.96 & & \multicolumn{2}{c}{15.10} \\
$P$ & & & 0.003 & & \multicolumn{2}{c}{0.000} \\
\hline
\end{tabular}

\section{Discussion and Conclusion}

The present study demonstrates that PTSD symptoms after exposure to earthquakes in a Chinese sample are associated with the 5-HTTLPR genetic polymorphism. Interestingly, among the study group with PTSD the S genotype was significantly higher when compared to controls, indicating an association between the S genotype and PTSD in this Chinese sample. Notably, our results are consistent with the hypothesis of a gene-by-environment $(G \times E)$ interaction that suggests variations in the 5-HTT gene influence psycho-pathological reactions to stressful experiences [13], such as earthquakes.

PTSD is an important post-disaster mental and behavioral disorder with high incidence and prevalence. In addition this disorder has a potentially long duration and poor efficacy characteristics that seriously affect clinical treatment [14]. The increase in natural disasters, war, terrorism, and other precipitating factors of PTSD in recent years has resulted in more research related to the disorder. For example, the occurrence of the Wenchuan earthquake in China's Sichuan Province on May 12, 2008, measuring 8.0 on the Richter scale, created well-deserved attention and needed research about PTSD [15]. The attention has been intensified over the last years due to the increased number of natural disasters occurring in provinces of China.

Currently, despite the considerable amount of research on PTSD and its pathogenesis, the contribution of distinct genetic factors remains unclear. Stress diathesis theories predict that an individual's sensitivity to stressful events depends on their genetic background [16], and such predictions are now increasingly supported by experimental evidence. Much of this research has been focused in the analysis of polymorphisms in single genes or individual sites. One polymorphism that receives increased attention over the years is the serotonin 
transporter gene polymorphism (5-HTTLPR). Heils et al. first cloned the human 5 -HTT coding gene, which is encoded by a single gene SLC6A4 and located on chromosome $17 \mathrm{q} 12$ region [17]. Although the 5-HT system has been shown to modulate mood, irritability, and sleep disorders [18], the exact mechanism by which 5-HT modulates PTSD symptoms is still unclear.

The 5-HTTLPR polymorphism occurs in the promoter region of the 5-HTT gene that results in two variations in humans, a short (S) and a long (L) allele. Specifically, the S allele results in a lower expression of the 5-HTT compared with the L allele [19]. In other words, in vitro experiments have shown that the L fragment allele has a higher transcriptional activity than the $\mathrm{S}$ allele. In addition, cultured brain cells with the LL genotype result in higher 5-HTT mRNA and protein expression than the SS genotype. Thus, changes in 5-HTT protein expression may impair the 5-HT system function and affect individual's emotion [20]. In particular, variations in the functional human polymorphism 5-HTTLPR have been hypothesized to affect risk for PTSD [21]. Interestingly, the 5-HTTLPR human polymorphism has been also associated with neuroticism [22] and increased vulnerability to affective disorders [23], in many but not all studies [24]. For example, the $S$ allele carriers, that have lower expression of 5-HTT, are more vulnerable to depression and suicidality in response to stressful events during adulthood than are individuals carrying the $\mathrm{L}$ allele, that have higher expression of 5-HTT [25].

With the aim of investigating the association between PTSD and the 5-HTTLPR polymorphism, we conducted an analysis to shed light on this interaction in a Chinese sample that had suffered from natural disasters in a context that has not been previously studied. By determining the genotype and allele frequency distribution among PTSD patients and healthy control subjects we demonstrate that a higher number of PTSD patients have the $S$ variant when compared to controls. This result suggests that the S allele of 5-HTTLPR is significantly associated with PTSD symptoms and is in line with the mechanistic model of the 5-HTTLPR polymorphism. This model of the human 5-HTTLPR polymorphism suggests that different transcription of the 5-HTT gene creates populations of individuals with lower expression of 5-HTT (S carriers) that exhibited more PTSD symptoms in relation to stressful life events than individuals with higher expression of 5-HTT (L carriers).

Kate et al. Prior studies have found that the 5-HTTLPR polymorphism in the promoter region of the serotonin transporter gene (SLC6A4) interacts with stressful life events to increase general risk for PTSD, 5-HTTLPR genotype modified the effect of emotional abuse on PTSD symptom severity. Participants with the low-expression SS genotype who were exposed to emotional abuse had significantly lower reexperiencing and arousal symptom severity scores [26]. Consistent with our results, when examined in a relatively homogenous sample with shared trauma and known prior levels of child and adult trauma, the 5-HTTLPR multimarker genotype may serve as a useful predictor of risk for PTSD-related symptoms in the weeks and months following the trauma [27]. Notably, previous 
studies have also shown an association of the 5-HTTLPR with different mental conditions; for example, the SS genotype often appears in patients with severe depression and anxiety disorders [28]. Furthermore, in a study of a large sample of twins it has been reported that individuals containing 5-HTTLPR SS or SL are more prone to depressive reactions of PTSD after stressful events [29]. Other study showed that the SS or SL allele carriers have a higher amygdala activity than the LL allele carriers when the individual encountered fear-related stimuli, and that the anxiety levels were lower in SL carriers when compared to LL carriers. Thus, these results suggest that the $S$ genotype of the 5-HTTLPR may prompt fear and anxiety related symptoms [30]. Furthermore, it has been also shown that individuals with two short alleles (SS or SL) of 5-HTT polymorphisms are more vulnerable to stress than those who have only one or two long alleles (LL) [31], indicating a higher susceptibility risk. Further, mutations in the 5-HTT gene can affect individuals' sensitivity to stress and may play a role in the extent or intensity of the stress reaction. Although few studies has investigated the association between the 5-HTTLPR and susceptibility to PTSD, it has been suggested that the observed amygdala activity improvement after fear exposure therapy is more likely to occur in SS and SL carriers when compared to LL carriers.

From our knowledge, there are not studies that had investigated the relationship between PTSD and 5-HTTLPR in a Chinese population but several lines of evidence suggested that this interaction exist in non-Chinese populations, where it has been demonstrated a correlation between the S allele carriers and PTSD symptoms [31]. The present study addresses this issue and, our results demonstrate that the association between PTSD and 5-HTTLPR also exists in the Chinese population. However, the small sample size of this study presents limitations in the study design and therefore we should be cautious with our own conclusions. For example, it is difficult to rule out the confounding effects of PTSD. Therefore, while the present study contributes importantly to the genetics and psychiatric disorders research in general and specifically in a Chinese context, these results could valuably be replicated, to establish further reliability and validity.

In sum, this epidemiological study provides evidence of a gene-by-environment interaction in a Chinese population, in which their genetic background moderates an individual's response to environmental insults. However, validation and replication of these results will allow us to begin to understand how certain vulnerability factors affects emotional functions to increase the risk for PTSD symptoms later in life. These results may help guide the choice of outcome measures in clinical studies and aid in the identification of molecular mechanisms that may confer vulnerability to PTSD disorders to help us to better categorize and manage this complex disease.

\section{Funded Projects}

2018 Hainan Provincial Key R \& D Project, No.: ZDYF2018133; 2017 Funded by the National Natural Science Foundation of China, project approval: 81760255. 


\section{Conflicts of Interest}

The authors declare no conflicts of interest regarding the publication of this paper.

\section{References}

[1] Harvey, P.D., Posner, K., Rajeevan, N., Yershova, K.V., Aslan, M. and Concato, J. (2018) Suicidal Ideation and Behavior in US Veterans with Schizophrenia or Bipolar Disorder. Journal of Psychiatric Research, 102, 216-222. https://doi.org/10.1016/j.jpsychires.2018.04.014

[2] Franklin, C.L., Raines, A.M., Chambliss, J.L., Walton, J.L. and Maieritsch, K.P. (2018) Examining Various Subthreshold Definitions of PTSD Using the Clinician Administered PTSD Scale for DSM-5. Journal of Affective Disorders, 234, 256-260. https://doi.org/10.1016/j.jad.2018.03.001

[3] Smith, N.B., Sippelb, L.M., Presseau, C., Rozek, D., Mota, N., Gordon, C., Horvath, M. and Harpaz-Rotemahi, I. (2018) Locus of Control in US Combat Veterans: Unique Associations with Posttraumatic Stress Disorder 5-Factor Model Symptom Clusters. Psychiatry Research, 268, 152-156. https://doi.org/10.1016/j.psychres.2018.07.015

[4] Mirhashem, R., Allen, H.C., Adams, Z.W., et al. (2017) The Intervening Role of Urgency on the Association between Childhood Maltreatment, PTSD, and Substance-Related Problems. Addictive Behaviors, 69, 98-103. https://doi.org/10.1016/j.addbeh.2017.02.012

[5] Seow, L.S.E., Ong, C., Mahesh, M.V., Sagayadevan, V., et al. (2016) A Systematic Review on Comorbid Post-Traumatic Stress Disorder in Schizophrenia. Schizophrenia Research, 176, 441-451. https://doi.org/10.1016/j.schres.2016.05.004

[6] Badour, C.L., Gros, D.F., Szafranski, D.D. and Acierno, R. (2016) Sexual Problems Predict PTSD and Depression Symptom Change Among Male OEF/OIF Veterans Completing Exposure Therapy. Psychiatry, 79, 403-417. https://doi.org/10.1080/00332747.2016.1142774

[7] Yeterian, J.D., Berke, D.S. and Litz, B.T. (2017) Psychosocial Rehabilitation after War Trauma with Adaptive Disclosure: Design and Rationale of a Comparative Efficacy Trial. Contemporary Clinical Trials, 61, 10-15. https://doi.org/10.1016/j.cct.2017.07.012

[8] Masheb, R.M., Ruser, C.B., Min, K.M., et al. (2018) Does Food Addiction Contribute to Excess Weight among Clinic Patients Seeking Weight Reduction? Examination of the Modified Yale Food Addiction Survey. Comprehensive Psychiatry, 84, 1-6. https://doi.org/10.1016/j.comppsych.2018.03.006

[9] Emerson, M.A., Moore, R.S. and Caetano, R. (2017) Association between Lifetime Posttraumatic Stress Disorder and Past Year Alcohol Use Disorder among American Indians/Alaska Natives and Non-Hispanic Whites. Alcoholism: Clinical and Experimental Research, 41, 576-584. https://doi.org/10.1111/acer.13322

[10] Verhey, R., Chibanda, D., Gibson, L., Brakarsh, J. and Seedat, S. (2018) Validation of the Posttraumatic Stress Disorder Checklist-5 (PCL-5) in a Primary Care Population with High HIV Prevalence in Zimbabwe. BMC Psychiatry, 18, 109. https://doi.org/10.1186/s12888-018-1688-9

[11] Alhalal, E., Ford-Gilboe, M., Wong, C. and AlBuhairan, F. (2017) Reliability and Validity of the Arabic PTSD Checklist Civilian Version (PCL-C) in Women Survivors of Intimate Partner Violence. Research in Nursing \& Health, 40, 575-585. 
https://doi.org/10.1002/nur.21837

[12] Reznikov, R., Rodriguez, F., et al. (2018) Prefrontal Cortex Deep Brain Stimulation Improves Fear and Anxiety-Like Behavior and Reduces Basolateral Amygdala Activity in a Preclinical Model of Posttraumatic Stress Disorder. Neuropsychopharmacology, 43, 1099-1106. https://doi.org/10.1038/npp.2017.207

[13] Giordano, N.A. and Bader, C. (2018) Complexity of the Relationships of Pain, Posttraumatic Stress, and Depression in Combat-Injured Populations: An Integrative Review to Inform Evidence-Based Practice. Worldviews on Evidence-Based Nursing, 15, 113-126. https://doi.org/10.1111/wvn.12274

[14] Mills, K.L., Christina, M., et al. (2018) The Long-Term Impact of Post Traumatic Stress Disorder on Recovery from Heroin Dependence. Journal of Substance Abuse Treatment, 89, 60-66. https://doi.org/10.1016/j.jsat.2018.04.001

[15] Hu, X., Cao, X., et al. (2016) Probable Post-Traumatic Stress Disorder and Its Predictors in Disaster-Bereaved Survivors: A Longitudinal Study after the Sichuan Earthquake. Archives of Psychiatric Nursing, 30, 192-197. https://doi.org/10.1016/j.apnu.2015.08.011

[16] Foa, E.B., McLean, C. and, Peterson, A. (2018) Motivation to Participate in PTSD Research-Reply. JAMA, 319, 2440-2441. https://doi.org/10.1001/jama.2018.4330

[17] Heils, A.R. and Lesch, M.K.P. (1997) The Human Serotonin Transporter Gene Polymorphism-Basic Research and Clinical Implications. Journal of Neural Transmission, 104, 1005-1014. https://doi.org/10.1007/BF01273314

[18] Murphy, S., Hansen, M., et al. (2018) Alternative Models of DSM-5 PTSD: Examining Diagnostic Implications. Psychiatry Research, 262, 378-383. https://doi.org/10.1016/j.psychres.2017.09.011

[19] Hou, L., Qi, Y., Sun, H., Wang, G., et al. (2018) Applying Ketamine to Alleviate the PTSD-Like Effects by Regulating the HCN1-Related BDNF. Progress in Neuro-Psychopharmacology \& Biological Psychiatry, 8, 313-321. https://doi.org/10.1016/j.pnpbp.2018.03.019

[20] Lee, D., Liverant, G., Lowmaster, S.E. Gradusc, J.L. and Sloan, D.M. (2014) PTSD and Reasons for Living: Associations with Depressive Symptoms and Alcohol Use. Psychiatry Research, 219, 550-555. https://doi.org/10.1016/j.psychres.2014.06.016

[21] Murkar, A., Kent, P.C, James, C. and Merali, Z. (2018) Gastrin-Releasing Peptide Attenuates Fear Memory Reconsolidation. Behavioural Brain Research, 347, 255-262. https://doi.org/10.1016/j.bbr.2017.11.037

[22] Thling, J., Simmons, C., Suliman, S. and Seedat, S. (2017) Trauma Type as a Conditional Risk Factor for Posttraumatic Stress Disorder in a Referred Clinic Sample of Adolescents. Comprehensive Psychiatry, 76, 138-146.

https://doi.org/10.1016/j.comppsych.2017.05.001

[23] Herz, N., Reuveni, I., et al. (2016) Neural Correlates of Attention Bias in Posttraumatic Stress Disorder. Clinical Neurophysiology, 127, 3268-3276. https://doi.org/10.1016/j.clinph.2016.07.016

[24] Broekman, B.F.P., Olff, M. and Boer, F. (2007) The Genetic Background to PTSD. Neuroscience and Biobehavioral Reviews, 31, 348-362. https://doi.org/10.1016/j.neubiorev.2006.10.001

[25] Aslihan, S., et al. (2010) A Prospective Study of Serotonin Transporter Gene Promoter (5-HTT Gene Linked Polymorphic Region) and Intron 2 (Variable Number of Tandem Repeats) Polymorphisms as Predictors of Trauma Response to Mild Physical Injury. DNA and Cell Biology, 29, 71-77. https://doi.org/10.1089/dna.2009.0936 
[26] Walsh, K., Uddin, M., Soliven, R., Wildman, D.E. and Bradley-Davino, B. (2014) Associations between the SS variant of 5-HTTLPR and PTSD among Adults with Histories of Childhood Emotional Abuse: Results from Two African American Independent Samples. Journal of Affective Disorders, 161, 91-96. https://doi.org/10.1016/j.jad.2014.02.043

[27] Mercer, K.B. (2012) Acute and Posttraumatic Stress Symptoms in a Prospective Gene x Environment Study of a University Campus Shooting. Archives of General Psychiatry, 69, 89-97. https://doi.org/10.1001/archgenpsychiatry.2011.109

[28] Tian, Y., Liu, H. and Guse, L. (2015) Association of Genetic Factors and Gene-Environment Interactions with Risk of Developing Posttraumatic Stress Disorder in a Case-Control Study. Biological Research for Nursing, 17, 364-372. https://doi.org/10.1177/1099800415588362

[29] Wang, Z., Dewleen, G., et al. (2011) The Relationship between Combat-Related Posttraumatic Stress Disorder and the 5-HTTLPR/rs25531 Polymorphism. Depression and Anxiety, 28, 1067-1073. https://doi.org/10.1002/da.20872

[30] Murrough, J.W., Huang, Y., et al. (2011) Reduced Amygdala Serotonin Transporter Binding in Posttraumatic Stress Disorder. Biological Psychiatry, 70, 1033-1038. https://doi.org/10.1016/j.biopsych.2011.07.003

[31] Sayin, A., Kucukyildirim, S., et al. (2010) A Prospective Study of Serotonin Transporter Gene Promoter (5-HTT Gene Linked Polymorphic Region) and Intron 2 (Variable Number of Tandem Repeats) Polymorphisms as Predictors of Trauma Response to Mild Physical Injury. DNA and Cell Biology, 29, 71-77. https://doi.org/10.1089/dna.2009.0936

\section{Highlights}

- Our results are consistent with the hypothesis of a gene-by-environment interaction.

- The S genotype was higher in the PTSD group when compared to controls.

- PTSD due to earthquakes in a Chinese sample is associated with the 5-HTTLPR. 